\title{
Neglected evidence in idiopathic pulmonary fibrosis and the importance of early diagnosis and treatment
}

\author{
Vincent Cottin ${ }^{1,2}$ and Luca Richeldi $i^{3,4}$
}

\begin{abstract}
Affiliations: ${ }^{1}$ Hospices Civils de Lyon, Hôpital Louis Pradel, Service de pneumologie - Centre de référence national des maladies pulmonaires rares, Lyon, and ${ }^{2}$ Université de Lyon, Université Claude Bernard Lyon 1 , INRA, UMR754 INRA-Vetagrosup EPHE IFR 128, Lyon, France. ${ }^{3}$ Academic Unit of Clinical and Experimental Sciences, Faculty of Medicine, University of Southampton, Southampton, and ${ }^{4}$ Southampton NIHR Respiratory Biomedical Research Unit, University Hospital Southampton, Southampton, UK.
\end{abstract}

Correspondence: L. Richeldi, Academic Unit of Clinical and Experimental Sciences, Faculty of Medicine, University of Southampton, Southampton General Hospital, Mailpoint 801, South Academic Block, Tremona Road, Southampton, S016 6YD, UK. E-mail: L.Richeldidsoton.ac.uk

ABSTRACT In idiopathic pulmonary fibrosis (IPF), some facts or concepts based on substantial evidence, whilst implicit for learned subspecialists, have previously been neglected and/or not explicitly formulated or made accessible to a wider audience. IPF is strongly associated with cigarette smoking and is predominantly a disease of ageing. However, its cause(s) remain elusive and, thus, it is one of the most challenging diseases for the development of novel effective and safe therapies. With the approval of pirfenidone for patients with mild-to-moderate IPF, an earlier diagnosis of IPF is a prerequisite for earlier treatment and, potentially, improvement of the long-term clinical outcome of this progressive and ultimately fatal disease. An earlier diagnosis may be achieved in IPF by promoting thin-slice chest high-resolution computed tomography screening of interstitial lung disease as a "by-product" of large-scale lung cancer screening strategies in smokers, but other techniques, which have been neglected in the past, are now available. Lung auscultation and early identification of "velcro" crackles has been proposed as a key component of early diagnosis of IPF. An ongoing study is exploring correlations between lung sounds on auscultation obtained using electronic stethoscopes and high-resolution computed tomography patterns.

0 @ERSpublications

Lung cancer screening and electronic recording of lung sounds might help early detection of idiopathic pulmonary fibrosis http://ow.ly/rScQU

\section{Introduction}

A recent review by CORDIER and COTTIN [1] addressed a number of important facts and concepts in idiopathic pulmonary fibrosis (IPF), largely based on circumstantial, but relatively strong, evidence that they stated may be "implicit for learned subspecialties but which have not been explicitly formulated and made accessible to a wider audience".

These concepts included the observation that, although the description of IPF is often spuriously credited to William Osler over a century ago, more precise descriptions of IPF only appeared in the medical literature in the second half of the 20th century, suggesting that IPF is in fact a relatively "recent" disease. This concept may also be linked to the fact that IPF is a direct consequence of the epidemics of cigarette smoking, such as lung cancer, which appeared during the first decades of the 20th century [2]. Although IPF is not

Received: Dec 062013 | Accepted: Dec 132013

Conflict of interest: Disclosures can be found alongside the online version of this article at err.ersjournals.com

Provenance: Publication of this peer-reviewed article was supported by InterMune International AG, Muttenz, Switzerland (article sponsor, European Respiratory Review issue 131).

Copyright OERS 2014. ERR articles are open access and distributed under the terms of the Creative Commons Attribution Non-Commercial Licence 3.0. 
presently included in the list of tobacco-associated lung diseases, the prevalence of IPF has, to a degree, paralleled that of lung cancer and chronic obstructive pulmonary disease (COPD). The frequency of smoking in IPF patients also parallels that in lung cancer and COPD $(\sim 60-80 \%)$. Smoking is not associated with IPF in all patients; however, there is a clear interaction between smoking, age and genetic background in the development of interstitial lung diseases (ILDs) [3, 4]. The impact of ageing and genetic background on the development of IPF is well illustrated by, among other facts, telomere shortening. Syndromes of short telomeres represent archetypal premature ageing syndromes of the lungs [5], and short telomeres have been found in $\sim 40 \%$ of patients with familial IPF and up to $25 \%$ of patients with sporadic IPF (fig. 1) [6]. Studies indicate that over two-thirds of IPF patients with telomerase mutations are smokers and that current and former smokers have shorter telomeres than age-matched controls $[7,8]$. For example, a typical case of IPF associated with a telomerase mutation may present as a person aged $<50$ years with macrocytosis, premature greying (eyebrows and hair roots) and fine inspiratory crackles on lung auscultation, with further investigation revealing the patient to have a telomerase reverse transcriptase (TERT) gene mutation and usual interstitial pneumonia (UIP) on lung biopsy [9].

\section{The importance of early diagnosis of IPF}

With the availability of therapies to slow disease progression, earlier diagnosis of IPF is of increasing importance and awareness of such presentations described above may raise diagnostic suspicion of the disease. The importance of an earlier diagnosis of IPF is relevant to any chronic, progressive, irreversible disorder but this becomes crucially important when effective treatments are available that can then be started without delay. A "window of opportunity" may exist during which time treatment can have optimal outcomes (fig. 2) [10]. Furthermore, delayed diagnosis of IPF is associated with increased costs in terms of investigations performed, and delayed referral can result in lower survival rates independent of disease severity or associated prognostic factors (fig. 3) [11, 12].

However, an early diagnosis in IPF can be problematic due to ambiguous diagnostic criteria. For example, according to the 2011 American Thoracic Society/European Respiratory Society/Japanese Respiratory Society/Latin American Thoracic Association guidelines, if high-resolution computed tomography (HRCT) scanning yields a radiological pattern that is not clearly IPF but is suggestive of IPF, i.e. possible IPF, the patient will need a surgical lung biopsy to confirm that they have definite IPF [13]. Increasingly, in an ageing population it is becoming difficult to perform surgical lung biopsy and, therefore, without a biopsy the patient is left with a diagnosis of suspected IPF. As in any other disease, in IPF it can be difficult to initiate treatment in the absence of a clear diagnosis. A solution may, in part, lie in adopting guidelines similar to those of the National Institute for Health and Care Excellence (NICE) in the UK [14]. The NICE guidance advocates that the clinician maintains a high level of suspicion for IPF in patients presenting with typical features such as bilateral inspiratory crackles ("velcro crackles") on chest auscultation and finger clubbing. Specifically, NICE recommends that healthcare professionals, particularly primary care physicians, should be aware of the symptoms of this disease to make sure patients have access to chest imaging (especially HRCT) and specialists, so that a prompt diagnosis can be made and appropriate IPF management can be initiated following a diagnosis of suspected IPF (table 1). It is also important to note that these same guidelines clearly state that patients with IPF may often present with normal lung function, in particular during the early stages of the disease.

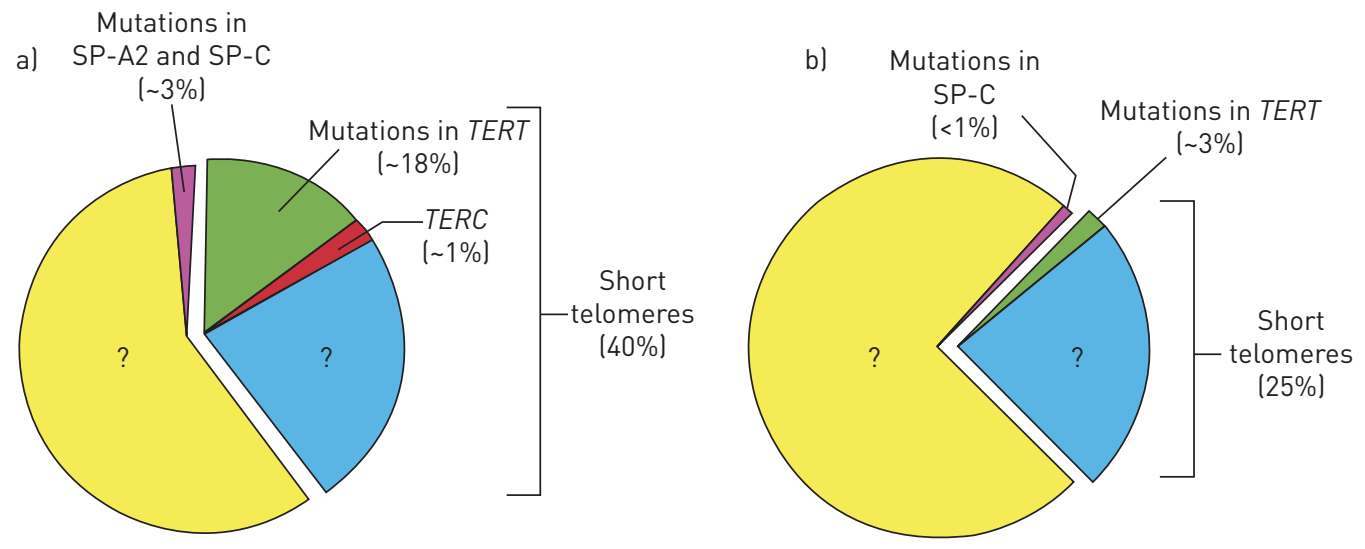

FIGURE 1 Telomere length and telomerase mutations in a) familial and b) sporadic idiopathic pulmonary fibrosis. SP: surfactant protein; TERT: telomerase reverse transcriptase; TERC: telomerase RNA component. Reproduced from [6] with permission from the publisher. 


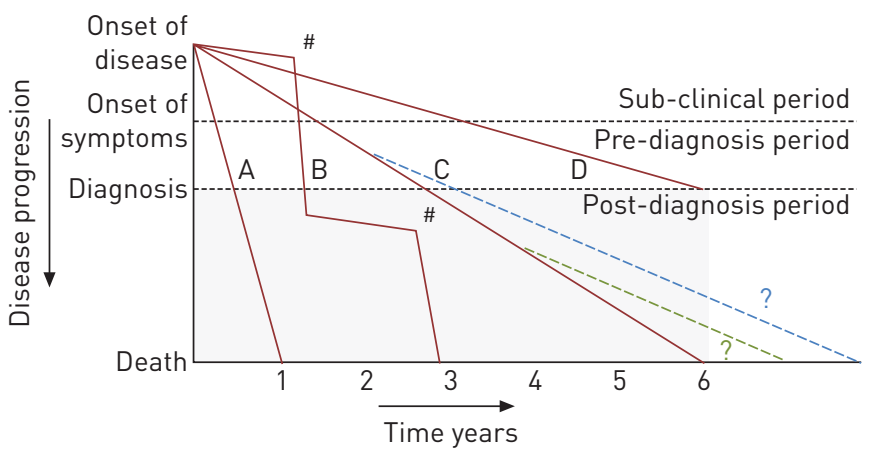

FIGURE 2 Earlier treatment of idiopathic pulmonary fibrosis (IPF) may impact the course of the disease. The red lines denote the various possible disease courses. The green dotted line indicates the anticipated hypothetical outcome in patients treated late in the course of disease and the blue dotted line indicates patients treated following early IPF diagnosis. A: fast decline without treatment; B: episodes of acute worsening; C: chronic progressive decline without treatment; D: slower decline without treatment. "\#: periods of relative stability interposed with periods of acute decline. Reproduced from [10] with permission from the publisher.

With the development and approval of new treatments for IPF, screening programmes are key components of earlier diagnosis. Currently, there are two realistic approaches for earlier identification of IPF: 1) recognising the value of velcro crackles at pulmonary auscultation through proactive education of primary care physicians; and 2) promoting the screening for IPF as a by-product of thin-slice, low-dose chest computed tomography (CT) screening for lung cancer in smokers and ex-smokers.

\section{Identifying velcro crackles for earlier diagnosis of IPF}

Although not specific for IPF, velcro crackles are one of the key elements for early diagnosis and are often present long before an IPF diagnosis is finally made. This topic has been reviewed recently by COTTIN and CORDIER [15], who proposed that assessment of velcro crackles on lung auscultation is a practical way to improve the earlier diagnosis of IPF. Fine crackles are easily recognised by clinicians and are characteristic of ILD. If bilateral fine crackles are present throughout the inspiratory time, persist after several deep breaths and remain present on numerous occasions several weeks apart in a subject aged $\geqslant 60$ years, this should raise the suspicion of IPF and lead to consideration of a HRCT scan of the chest [15].

Paradoxically, earlier referral of suspected IPF for HRCT diagnosis raises an issue in that criteria for confident HRCT diagnosis requires the presence of honeycombing, which is often a late sign, mostly occurring in advanced disease [13]. CORDIER and COTTIN [1] have proposed that lung biopsy could, therefore, be discussed with patients with suspected IPF during the "honeymoon" of early IPF, i.e. when velcro crackles can be heard on lung auscultation and before the appearance of honeycombing on HRCT. First, however, there is the need for well-designed, prospective studies that can re-appraise the value of lung auscultation for the early diagnosis of IPF.

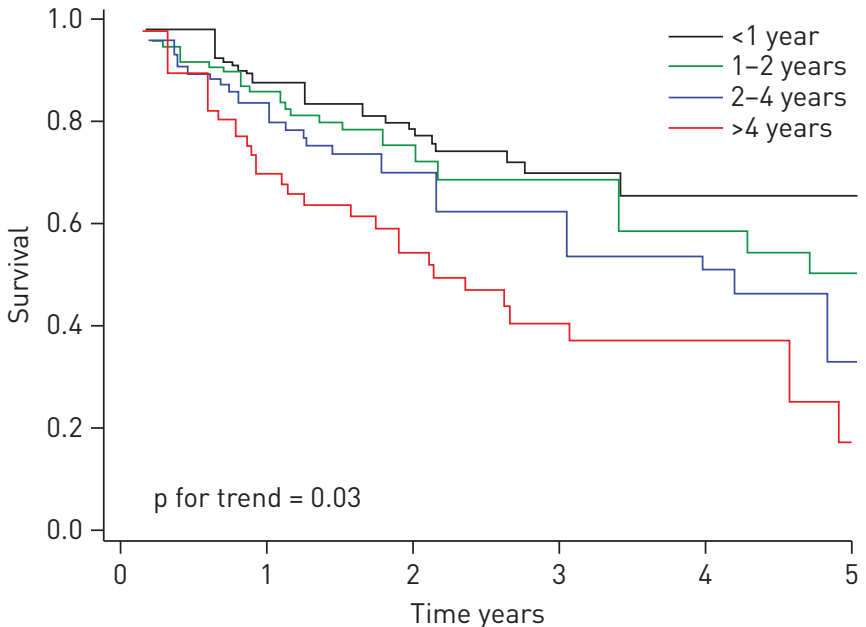

FIGURE 3 Earlier treatment of idiopathic pulmonary fibrosis may improve survival rates. Reproduced from [12] with permission from the publisher. 
TABLE 1 Awareness of clinical features of idiopathic pulmonary fibrosis (IPF)

Be aware of IPF when assessing a patient with the clinical features listed below and when considering requesting a chest radiograph or referring to a specialist:

Age $>50$ years

Persistent breathlessness on exertion

Persistent cough

Bilateral inspiratory crackles when listening to the chest

Clubbing of the fingers

Normal spirometry or impaired spirometry, usually with a restrictive pattern

Information from [14].

\section{Correlation between lung sounds and HRCT pattern in ILD: design of a prospective study}

During the 2013 European Respiratory Society Annual Congress in Barcelona, Spain, L. Richeldi presented the session "Importance of early diagnosis and treatment", the design of a prospective study, which aims to identify potential correlations between acoustic patterns obtained using electronic stethoscopes and HRCT images. The population in this study includes patients undergoing chest HRCT for any indication and the collection of demographic data, reasons for requesting chest HRCT, medical history (smoking, exposures and drugs), respiratory signs and symptoms, and the diagnosis is eventually made after complete evaluation. An electronic stethoscope capable of recording the auscultation is used to listen to the chest at six auscultation points, as marked by metallic marks recognisable on HRCT scans (fig. 4). These metallic marks serve as a point of reference denoting which part of the lung is generating the acoustic pattern in question. Lung sounds and the presence of velcro crackles can be recorded first by the electronic stethoscope and then the time, frequency and intensity of these sound waves can be objectively quantified and imaged threedimensionally through the application of specifically designed software. The recording of sound files may enable a comparison of lung sounds over time and, ultimately, the creation of a spectrum of predicted values. It is anticipated that results from this study will be available in 2014 .

\section{Low-dose HRCT screening for IPF}

Large-scale screening programmes are currently under consideration for lung cancer and the adoption of such a scheme for IPF, or as a by-product of lung cancer screening, may also be used to diagnose and treat IPF earlier, taking advantage of similarities in risk factors for IPF and lung cancer, e.g. age and tobacco smoking. Jin et al. [16] conducted low-dose CT screening for lung cancer in cigarette smokers with the aim of determining the prevalence of interstitial lung abnormalities at initial CT examination and the rate of progression of interstitial lung abnormalities on 2-year follow-up CT images. Interstitial lung abnormalities were observed in $9.7 \%$ of patients and $2.1 \%$ of this total was due to fibrotic abnormalities. An association with current smoking and number of pack-years with interstitial lung abnormalities was also observed. At the 2-year follow-up CT scan, fibrotic interstitial lung abnormalities had progressed in $37 \%$ of patients, including to a typical pattern of UIP/IPF in some, and regressed in none. Although the study screened patients for lung cancer, it highlights the potential of such screening strategies to detect early IPF as a byproduct of cancer screening [17].

FIGURE 4 Placement of metallic markers to correlate location of chest sound and high-resolution computed tomography image.

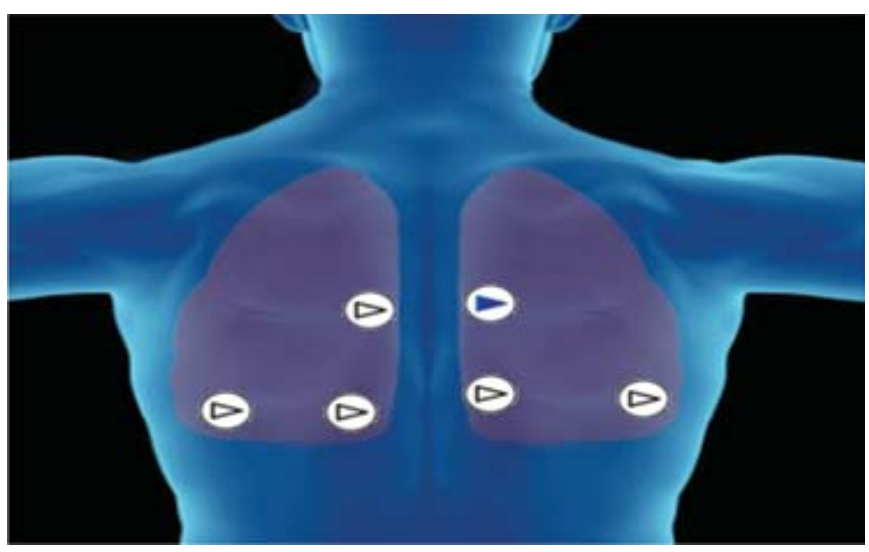




\section{Conclusion}

An earlier diagnosis of IPF is a prerequisite for earlier treatment and, potentially, improvement of the longterm clinical outcome of this progressive and ultimately fatal disease. If IPF is suspected, diagnosis can be challenging but a multidisciplinary approach involving a pulmonologist, radiologist and pathologist who are expert in ILD has been shown to improve the accuracy of IPF diagnosis. The implementation of a screening programme for IPF in clinical practice presents several challenges and the validation of adequate diagnostic studies are required in the future; both into the adoption of velcro crackles in the diagnostic process and the long-term clinical utility of detecting early IPF in large scale, thin-slice HRCT screening programmes for lung cancer.

\section{Acknowledgements}

This article is based on the proceedings of a satellite symposium held at the 2013 European Respiratory Society Annual Congress (Barcelona, Spain), which was sponsored by InterMune. We were assisted in the preparation of the article by IntraMed International (Milan, Italy), funded by InterMune.

\section{References}

1 Cordier JF, Cottin V. Neglected evidence in idiopathic pulmonary fibrosis: from history to earlier diagnosis. Eur Respir J 2013; 42: 916-923.

2 Doll R, Peto R, Boreham J, et al. Mortality in relation to smoking: 50 years' of observations on male British doctors. BMJ 2004; 328: 1519.

3 Taskar VS, Coultas DB. Is idiopathic pulmonary fibrosis an environmental disease? Proc Am Thorac Soc 2006; 3: 329-328.

4 Steele MP, Speer MC, Loyd JE, et al. Clinical and pathologic features of familial interstitial pneumonia. Am J Respir Crit Care Med 2005; 172: 1146-1152.

Armanios M. Syndromes of telomere shortening. Annu Rev Genomics Hum Genet 2009; 10: 45-61.

Garcia CK. Idiopathic pulmonary fibrosis: update on genetic discoveries. Proc Am Thorac Soc 2011; 8: 158-162.

Diaz de Leon A. Telomere lengths, pulmonary fibrosis and telomerase (TERT) mutations. PLoS One 2010; 5: el0680.

8 Valdes AM, Andrew T, Gardner JP, et al. Obesity, cigarette smoking, and telomere length in women. Lancet 2005; 366: 662-664.

9 Chambers DC, Clarke BE, McGaughran J, et al. Lung fibrosis, premature graying, and macrocytosis. Am J Respir Crit Care Med 2012; 186: e8-e9.

10 Ley B, Collard HR, King TE Jr. Clinical course and prediction of survival in idiopathic pulmonary fibrosis. Am J Respir Crit Care Med 2011; 183: 431-440.

11 Palfrey S. Daring to practice low-cost medicine in a high-tech era. N Engl J Med 2011; 364: e21.

12 Lamas DJ, Kawut SM, Bagiella E, et al. Delayed access and survival in idiopathic pulmonary fibrosis: a cohort study. Am J Respir Crit Care Med 2011; 184: 842-847.

13 Raghu G, Collard HR, Egan JJ, et al. An official ATS/ERS/JRS/ALAT statement: idiopathic pulmonary fibrosis: evidence-based guidelines for diagnosis and management. Am J Respir Crit Care Med 2011; 183: 788-824.

14 National Institute for Health and Care Excellence. Idiopathic pulmonary fibrosis. The diagnosis and management of suspected idiopathic pulmonary fibrosis. 2013. www.nice.org.uk/nicemedia/live/14183/64124/64124.pdf Date last accessed: October 25, 2013.

15 Cottin V, Cordier JF. Velcro crackles: the key for early diagnosis of idiopathic pulmonary fibrosis. Eur Respir J 2012; 40: 519-521.

16 Jin GY, Lynch D, Chawla A, et al. Interstitial lung abnormalities in a CT lung cancer screening population: prevalence and progression rate. Radiology 2013; 268: 563-571.

17 Cordier JF, Cottin V, Khouatra C, et al. Screening for lung cancer and idiopathic pulmonary fibrosis: killing two birds with one stone. Radiology 2014 [In press] 